\title{
CONSTRUÇÃO E VALIDAÇÃO DE INSTRUMENTO PARAAVALIAÇÃO DO CONHECIMENTO SOBRE ESTOMIAS INTESTINAIS DE ELIMINAÇÃO
}

Ana Karine da Costa Monteiro, Moniki de Oliveira Barbosa Campos, Jesusmar Ximenes Andrade, Elaine Maria Leite Rangel Andrade

Objetivo: descrever a construção e validação de instrumento para avaliação do conhecimento de alunos de Graduação em Enfermagem e enfermeiros sobre o cuidado de Enfermagem no perioperatório de cirurgias geradoras de estomias intestinais de eliminação. Metodologia: pesquisa metodológica realizada após aprovação do Comitê de Ética em Pesquisa para construção dos itens a partir da literatura e diretrizes internacionais e validação de conteúdo e aparência por oito especialistas/juízes em Estomaterapia. Para as análises, consideraram-se Índice de Validade de Conteúdo \a 80,0\% e Coeficiente Kappa de Fleiss $\geq$ a 70\%. Resultados: A versão final do instrumento foi constituída de 39 itens, divididos em 7 domínios, com IVC global de $94 \%$ e Coeficiente Kappa de Fleiss perfeito 1,00, após segunda rodada. Conclusão: Foi possível construir e validar o instrumento que poderá evidenciar lacunas do conhecimento existentes sobre o cuidado de Enfermagem no perioperatório das cirurgias geradoras de estomias intestinais de eliminação.

Descritores: Estomia; Estudos de Validação; Enfermagem.

\section{CONSTRUCTION AND VALIDATION OF AN INSTRUMENT FOR EVALUATION OF KNOWLEDGE ABOUT INTESTINAL ELIMINATION}

Objective: To describe the construction and validation of an instrument for assessing the knowledge of undergraduate nursing students and nurses about nursing care in the perioperative period of surgery to generate intestinal elimination ostomy. Methodology: Methodological research conducted after approval by the Research Ethics Committee for construction of items from the literature and international guidelines and validation of content and appearance by eight stomatherapy experts / judges. For the analyzes, we considered Content Validity Index $\geq 80.0 \%$ and Kappa de Fleis Coefficient $\geq 70 \%$. Results: The final version of the instrument consisted of 39 items, divided into 7 domains, with overall CVI of 94\% and Perfect Fleiss Kappa Coefficient 1.00, after the second round. Conclusion: It was possible to build and validate the instrument that may highlight gaps in knowledge about nursing care in the perioperative period of surgery that generates elimination intestinal ostomy. Descriptors: Ostomy; Validation Studies; Nursing.

\section{CONSTRUCCIÓNY VALIDACIÓN DE INSTRUMENTOS PARA LA EVALUACIÓN DEL CONOCIMIENTO SOBRE LAS ESTANCIAS INTESTINALES DE ELIMINACIÓN}

Objetivo: Describir la construcción y validación de un instrumento para evaluar el conocimiento de los estudiantes de enfermería y las enfermeras sobre la atención de enfermería en el período perioperatorio de cirugía para generar ostomía de eliminación intestinal. Metodología: Investigación metodológica realizada después de la aprobación del Comité de Ética de Investigación para la construcción de artículos de la literatura y las directrices internacionales y la validación del contenido y la apariencia por ocho jueces / expertos en estoterapia. Para los análisis, consideramos el índice de validez de contenido $\geq 80.0 \%$ y el coeficiente de Kappa de Fleis $\geq 70 \%$. Resultado: La versión final del instrumento consistió en 39 items, divididos en 7 dominios, con un IVC general del 94\% y el coeficiente Perfect Fleiss Kappa 1.00, después de la segunda ronda. Conclusión: fue posible construir y validar el instrumento que puede resaltar lagunas en el conocimiento sobre el cuidado de enfermería en el período perioperatorio de la cirugía que genera la eliminación de la ostomía intestinal.

Descriptores: Estomía; Estudios de Validación; Enfermería. 


\section{INTRODUÇÃO}

As malformações congênitas, doenças (câncer de cólon e reto) ou traumas (acidentes de trânsito, arma de fogo, arma branca ou objetos perfurantes) no intestino são condições que requerem intervenção cirúrgica para confecção de estomias intestinais de eliminação (colostomia e ileostomia), permitindo a saída de fezes e flatos ${ }^{(1)}$.

Após a confecção das estomias intestinais de eliminação, as pessoas estomizadas vivenciam alterações físicas, psicológicas, sociais e espirituais, demandando cuidado de Enfermagem que requer conhecimentos específicos, habilidades e suprimentos adequados (equipamentos coletores e adjuvantes) para atender às necessidades humanas básicas afetadas pelo adoecimento e orientação para o autocuidado (2).

Para atender esta demanda de cuidados, é necessária formação do enfermeiro sobre o perioperatório de cirurgias geradoras de estomias intestinais de eliminação, na Graduação, Pós- Graduação Latu Senso em Cursos de Especialização em Enfermagem em Estomaterapia e Programas de Educação Permanente nos serviços de saúde, que possam fornecer capacitação técnica e científica e aumentar o conhecimento e a confiança dos alunos de Enfermagem e enfermeiros no cuidado de pessoas no perioperatório de cirurgias geradoras de estomias intestinais de eliminação (3-5).

No Brasil, desde 1990, há empenho para esta formação específica, sendo o primeiro Curso em Enfermagem em Estomaterapia realizado pela Escola de Enfermagem da Universidade de São Paulo e até 2013, o referido Curso tinha formado cerca de 300 estomaterapeutas, de quase todos os Estados do pais e de alguns países da América Latina (Argentina, $\mathrm{Pa}$ raguai, Uruguai e Colômbia) (6). Atualmente, há cerca de 20 desses Cursos credenciados no país e outros em credenciamento ${ }^{(7)}$.

Entretanto, apesar de todo este empenho para formação de estomaterapeutas no país, estudos ainda mostram que alunos de Graduação em Enfermagem e enfermeiros têm pouco conhecimento sobre o cuidado de pessoas no perioperatório de cirurgias geradoras de estomias intestinais de eliminação. Algumas destas pesquisas que mensuraram o conhecimento de alunos de Graduação em Enfermagem e enfermeiros sobre estomias intestinais de eliminação utilizaram abordagem qualitativa ${ }^{(3,5)}$. No exterior e no Brasil, outras pesquisas realizadas mensuraram qualidade de vida ${ }^{(8)}$ e cuidados da estomia ${ }^{(9-10)}$. Recentemente, Estados Unidos e Irã validaram instrumentos para avaliar conhecimento de enfermeiros sobre estomias intestinais de eliminação (4.11). Mas, nenhum destes instrumentos possibilitava avaliação do conhecimento de graduandos de Enfermagem e enfermeiros sobre o cuidado no perioperatório de cirurgias geradoras de estomias intestinais de eliminação.
Os instrumentos identificados são genéricos, sendo necessárias outras pesquisas que construam e validem instrumentos que permitam avaliar o conhecimento de graduandos de Enfermagem e enfermeiros sobre o cuidado de Enfermagem no perioperatório de cirurgias geradoras de estomias intestinais de eliminação construídos a partir de Diretrizes internacionais ${ }^{(12-13)}$ e literatura nacional sobre a temática ${ }^{(6,14)}$.

Investigações desta natureza podem contribuir para identificação de lacunas do conhecimento de alunos de Graduação em Enfermagem e enfermeiros sobre o cuidado a pessoa no perioperatório de cirurgias geradoras de estomias intestinais de eliminação, produção de dados, redirecionamento do processo de ensino-aprendizagem acerca da temática para o cuidado de Enfermagem individualizado e sistemático, livre de riscos que a falta de conhecimento pode ocasionar.

Diante do exposto, o presente estudo teve como objetivo descrever a construção e validação de instrumento para avaliação do conhecimento de alunos de Graduação em Enfermagem e enfermeiros sobre o cuidado de Enfermagem no perioperatório de cirurgias geradoras de estomias intestinais de eliminação.

\section{METODOLOGIA}

\section{Tipo de estudo}

Trata-se de pesquisa metodológica, por lidar com desenvolvimento, validação e avaliação de instrumentos e métodos de investigação ${ }^{(15)}$

\section{Participantes da pesquisa}

Para validação foram convidados juízes, conforme os seguintes critérios de inclusão: ter titulação mínima de especialista em Estomaterapia, pelo menos um ano de experiência clínica e produção científica na área nos últimos cinco anos.

Participaram da primeira etapa oito especialistas/juizes, e na segunda etapa, cinco.

\section{Local do estudo}

Este estudo foi realizado em uma capital do nordeste brasileiro.

\section{Coleta de dados}

Realizada no período de março a julho de 2014, em duas fases: construção dos itens do instrumento e validação de conteúdo e aparência como proposto na literatura ${ }^{(16)}$.

A construção dos itens do instrumento para avaliação do conhecimento de alunos de Graduação em Enfermagem e enfermeiros sobre o cuidado de Enfermagem no perioperatório de cirurgias geradoras de estomias intestinais de eliminação foi feita a partir de Diretrizes internacionais ${ }^{(12-13)}$ e da literatura 
científica nacional sobre o cuidado de Enfermagem no perioperatório das cirurgias geradoras de estomias intestinais de eliminação ${ }^{(6,14)}$.

Quarenta e três itens foram construídos e divididos em nove domínios: Conceito (itens 1, 2 e 3), Indicação (item 4), Classificação (itens 5 e 6), Assistência de Enfermagem no Período Pré-Operatório (itens 7 a 17), Pós-Operatório Imediato (itens 18 a 32), Mediato (itens 34 a 37), Tardio (itens 38, 40, 41 e 42), Complicações Imediatas e Tardias (itens 33 e 43) e Direitos da Pessoa com Estomia (item 39). Para responder os itens do instrumento existiam três opções: Verdadeiro (V) no caso de concordar com o item, Falso (F) no caso de discordar do item e Não Sei (NS) no caso de não saber o item. Para cada acerto foi atribuido um ponto e o escore total do instrumento correspondeu à soma de todos os acertos, e não foram atribuidos pontos para respostas incorretas e Não Sei. O desempenho foi considerado satisfatório quando o escore total de acerto no instrumento foi igual ou maior a $80 \%{ }^{(15)}$.

A validação de conteúdo e aparência foi realizada para verificar informações sobre a representatividade e clareza de cada item do instrumento ${ }^{(17)}$. Oito especialistas/juízes foram selecionados por intencionalidade para participar do processo de validação, considerando os seguintes critérios de inclusão: ter titulação mínima de especialista em Estomaterapia, pelo menos um ano de experiência clínica e produção científica na área especializada nos últimos cinco anos. Todos os especialistas/juízes aceitaram participar da validação do instrumento. Este número de especialistas/juizes atende ao que a literatura recomenda, consistindo no mínimo de três e máximo de 10 (17).

Para realizar a validação cada especialista/juiz recebeu por e-mail uma carta convite composta por: objetivos do estudo, Termo de Consentimento Livre e Esclarecido (TCLE) em duas vias para assinatura em caso de aceite, questionário de caracterização dos especialistas/juízes, descrição e orientação quanto ao preenchimento do formulário de resposta sobre cada item ${ }^{(17)}$.

Os itens do instrumento foram classificados segundo as análises de conteúdo e aparência. O primeiro foi avaliado por meio de uma escala Likert de quatro pontos para indicar se o conteúdo de cada item do instrumento era "irrelevante", "um pouco relevante", "bastante relevante" ou "extremamente relevante" (17). Caso a avaliação do especialista/juiz fosse irrelevante ou pouco relevante, o mesmo deveria justificá-la. Quanto à clareza, os itens do instrumento foram avaliados de acordo com as seguintes alternativas: "sim", o item está claro; e "não", o item não está claro. Caso a avaliação do especialista/juiz fosse à segunda alternativa, o mesmo deveria justificá-la. Os juizes também foram questionados sobre os domínios e a adequação/ pertinência em relação aos itens componentes.

\section{Procedimentos de análise dos dados}

A análise do grau de relevância foi realizada por meio do cálculo do Índice de Validade de Conteúdo para cada item do instrumento e foram considerados validados os itens que obtiverem Índice de Validade de Conteúdo maior ou igual a $80,0 \%{ }^{(15)}$. Os itens que obtiveram Índice de Validade de Conteúdo inferior a 80,0\% foram excluídos ${ }^{(16)}$. Para mensuração do nível de concordância e consistência dos juízes em relação à clareza foi calculada para cada item do instrumento o Coeficiente Kappa de Fleiss e foram considerados validados os itens que obtiveram Coeficiente Kappa de Fleiss maior ou igual a 70,0\%(18). Os itens que obtiveram Kappa de Fleiss menor do que 70,0\% foram reformulados de acordo com as sugestões dadas pelos especialistas/juizes na primeira etapa de validação e nova etapa foi realizada até que o mesmo atingisse o valor esperado. No entanto, ressalta-se que a calculadora online ${ }^{(19)}$ utilizada para o cálculo do Coeficiente Kappa de Fleiss (http://justus.randolph.name/kappa) considera apenas aqueles especialistas/juizes que responderam todos os itens do instrumento quanto ao critério clareza. Então, foram descartados para este cálculo os especialistas/juízes que deixaram itens em branco.

\section{Procedimentos éticos}

O estudo cumpriu os requisitos formais da legislação nacional e internacional para a realização de pesquisas envolvendo seres humanos após aprovação do Comitê de Ética em Pesquisa, Protocolo no 22822314.2.0000.5214.

\section{RESULTADOS}

Dos especialistas/juízes que participaram da validação, todos $(n=8)$ eram do sexo feminino, com idade entre 37 e 61 anos, representada por média de 50,8 (DP=9,6) anos. Quanto a qualificação profissional, 3 (37,5\%) possuíam mestrado, 6 (75,0\%) atuavam na docência e tinham em média 15,5 $(\mathrm{DP}=13,4)$ anos de experiência em Estomaterapia, sendo o menor tempo um ano e o maior de 31. A média de publicações nos últimos cinco anos em Estomaterapia pelos especialistas/juízes foi de 5,6 (DP=8,6) artigos.

O Índice de Validade de Conteúdo global do instrumento foi de $94,0 \%$, sendo que 27 itens obtiveram Índice de Validade de Conteúdo 100,0\% e 14 itens 86,0\%. O Índice de Validade de Conteúdo de dois itens "presença de filtro no equipamento coletor" (item 5.3) e "equipamento coletor de uma e duas peças" (item 6.3) obtiveram Índice de Validade de Conteúdo abaixo de $80,0 \%$, o que acarretou a exclusão deles. Conforme sugestões dos especialistas/juízes, dois itens foram unidos, referentes à classificação das estomias em temporárias 
e definitivas e optou-se em retirar a avaliação da estomia por instrumento validado, até o momento inexistente no Brasil.

O Coeficiente Kappa de Fleiss de 10 itens foi 0,99 (itens 10 a 13, 20, 24, 28,41 a 43) e de 33 itens foi abaixo de 0,43 (itens la 9, 14 a 19, 21 a 23, 25 a 27, 29 a 40) na primeira etapa. Enquanto que, na segunda etapa o Coeficiente Kappa de Fleiss obteve resultado perfeito $(1,00)$

A versão final do instrumento foi constituída de 39 itens, divididos em 7 domínios: Conceito (itens 1, 2 e 3), Indicação (item 4), Classificação (item 5), Assistência de Enfermagem no Período Pré-Operatório (itens 6 a 16), Assistência de Enfermagem no Período Pós-Operatório Imediato (itens 7 a 32), Assistência de Enfermagem no Período Pós-Operatório Mediato (itens 33 a 35), Assistência de Enfermagem no Período Pós-Operatório Tardio (itens 36 a 39).

O Índice de Validade de Conteúdo e o Coeficiente Kappa de Fleiss de cada item do instrumento são apresentados na Tabela 1,2 e 3.

Tabela 1 - Distribuição do Índice de Validade de Conteúdo e Coeficiente Kappa de Fleiss para os domínios Conceito, Indicação e Classificação, segundo a avaliação dos especialistas/ juízes na primeira e segunda etapa. Teresina, 2014.

\begin{tabular}{lccc}
\multicolumn{1}{c}{ Itens } & IVC* & Kappa** & Kappa*** \\
\hline D1. Conceito & & & \\
\hline 1.0 Estomias & 0,86 & 0,33 & 0,99 \\
1.1 Colostomias & 0,86 & $-0,06$ & 0,99 \\
\hline 1.2 Ileostomias & 0,86 & $-0,06$ & 0,99 \\
\hline D2. Indicação & 1,00 & 0,06 & 0,99 \\
\hline D3. Classificação & & & \\
3.0 Temporáriasa & 1,00 & $-0,20$ & 0,99 \\
3.1 Definitivasa & 1,00 & $-0,20$ & 0,99 \\
*IVC primeira etapa *Kappa primeira etapa ${ }^{* * * K a p p a ~ s e g u n d a ~ e t a p a ~}$ & unido.
\end{tabular}

Tabela 2 - Distribuição do Índice de Validade de Conteúdo e Coeficiente Kappa de Fleiss para os Domínios Assistência de Enfermagem no Período Pré-operatório e Pós-operatório imediato, segundo a avaliação dos especialistas/juízes na primeira e segunda etapa. Teresina, 2014.

\begin{tabular}{|c|c|c|c|}
\hline \multirow{2}{*}{$\begin{array}{l}\text { Itens } \\
\text { D4 Assistência de Enfermagem } \\
\text { no Periodo Pré-operatório }\end{array}$} & \multirow[t]{2}{*}{ IVC* } & Kappa** & \multirow[t]{2}{*}{ Kappa*** } \\
\hline & & & \\
\hline 4.0 Consulta pré-operatório & 1,00 & $-0,06$ & 0,99 \\
\hline 4.1 Confecção do estoma & 0,86 & 0,33 & 0,99 \\
\hline 4.2 Características do estoma & 0,86 & 0,99 & 0,99 \\
\hline $\begin{array}{l}4.3 \text { Equipamento coletor e adju- } \\
\text { vantes }\end{array}$ & 0,86 & 0,99 & 0,99 \\
\hline 4.4 Impactona sexualidade & 1,00 & 0,99 & 0,99 \\
\hline
\end{tabular}

\begin{tabular}{|c|c|c|c|}
\hline 4.5 Demarcação do estoma & 1,00 & 0,99 & 0,99 \\
\hline 4.6 Posicionamento do estoma & 0,86 & $-0,20$ & 0,99 \\
\hline 4.7 Demarcação e complicações & 1,00 & $-0,20$ & 0,99 \\
\hline $\begin{array}{l}\text { 4.8 Profissional habilitado para } \\
\text { demarcação }\end{array}$ & 1,00 & 0,33 & 0,99 \\
\hline 4.9 Localização do estoma & 0,86 & 0,33 & 0,99 \\
\hline 4.10 Teste de sensibilidade & 1,00 & $-0,06$ & 0,99 \\
\hline \multicolumn{4}{|l|}{$\begin{array}{l}\text { D5 Assistência de Enfermagem } \\
\text { no Pós-operatório Imediato }\end{array}$} \\
\hline $\begin{array}{l}\text { 5.0 Aplicação do equipamento } \\
\text { coletor }\end{array}$ & 1,00 & $-0,06$ & 0,99 \\
\hline 5.1 Avaliação do estoma & 0,86 & 0,33 & 0,99 \\
\hline $\begin{array}{l}\text { 5.2 Características do equipa- } \\
\text { mento coletor }\end{array}$ & 1,00 & 0,99 & 0,99 \\
\hline 5.3 Filtrob & 0,71 & $-0,20$ & - \\
\hline 5.4 Edema no estoma & 1,00 & $-0,20$ & 0,99 \\
\hline 5.5 Cor do estoma & 1,00 & $-0,20$ & 0,99 \\
\hline 5.6 Forma do estoma & 1,00 & 0,99 & 0,99 \\
\hline 5.7 Saida de flatos & 1,00 & 0,33 & 0,99 \\
\hline 5.8 Primeiras eliminações & 1,00 & $-0,06$ & 0,99 \\
\hline 5.9 Aspectos do efluente & 0,86 & $-0,06$ & 0,99 \\
\hline
\end{tabular}

${ }^{*} \mathrm{IVC}$ primeira etapa *Kappa primeira etapa ***Kappa segunda etapa: unido; b: excluido.

Tabela 3 - Distribuição do Índice de Validade de Conteúdo e do Coeficiente Kappa de Fleiss para os Domínios Pós-operatório Mediato, Tardio, Complicações imediatas e tardias e Direitas da pessoa com estoma, segundo a avaliação dos especialistas/juizes na primeira e segunda etapa. Teresina, 2014.

\begin{tabular}{|c|c|c|c|}
\hline \multirow{2}{*}{$\begin{array}{l}\text { Itens } \\
\text { D6 Assistência de Enfermagem } \\
\text { no Pós-operatório Mediato }\end{array}$} & IVC* & \multicolumn{2}{|c|}{ Карра ${ }^{* *}$ Карра $a^{* * *}$} \\
\hline & & & \\
\hline 6.0 Diâmetro do estoma & 1,00 & $-0,20$ & 0,99 \\
\hline 6.1 Barreira protetora da pele & 0,86 & $-0,06$ & 0,99 \\
\hline $\begin{array}{l}6.2 \text { Escolha do equipamento co- } \\
\text { letor }\end{array}$ & 1,00 & $-0,06$ & 0,99 \\
\hline $\begin{array}{l}6.3 \text { Equipamento de uma ou } \\
\text { duas peçasb }\end{array}$ & 0,71 & $-0,06$ & - \\
\hline $\begin{array}{l}\text { D7 Assistência de Enfermagem } \\
\text { no Pós-operatório tardio }\end{array}$ & & & \\
\hline 7.0 Alta hospitalar & 1,00 & $-0,06$ & 0,99 \\
\hline 7.1 Referenciamento do cliente & 0,86 & $-0,06$ & 0,99 \\
\hline $\begin{array}{l}7.2 \text { Associações dos estomiza- } \\
\text { dos }\end{array}$ & 1,00 & 0,33 & 0,99 \\
\hline 7.3 Irrigação & 1,00 & 0,33 & 0,99 \\
\hline
\end{tabular}




$\begin{array}{llll}\text { 8.0 Complicações imediatas } & 0,86 & 0,99 & 0,99 \\ \text { 8.1 Complicações tardias } & 1,00 & 0,99 & 0,99\end{array}$

D9 Direitos da pessoa com estoma

9.0 Portaria $n^{\circ} 400 \quad 1,00 \quad 0,99 \quad 0,99$

${ }^{*}$ IVC primeira etapa *Kappa primeira etapa ***Kappa segunda etapaa: unido; ${ }^{\text {b: }}$ excluido.

\section{DISCUSSÃO}

Realizou-se construção e validação de conteúdo e aparência do instrumento para avaliação do conhecimento de Graduandos de Enfermagem e enfermeiros sobre o cuidado de Enfermagem no perioperatório de cirurgias geradoras de estomias intestinais de eliminação. Neste estudo, o Índice de Validade de Conteúdo global do instrumento foi de 94,0\% e o Coeficiente Kappa de Fleiss 1,00. Portanto, na avaliação dos especialistas/juizes que participaram deste estudo, o instrumento foi considerado validado no que diz respeito ao conteúdo e aparência. No entanto, este tipo de validação é limitado por ser subjetivo e não possibilitar a identificação de itens que possam ter sido omitidos do instrumento durante a sua construção ${ }^{(17)}$. Outro estudo realizado com esta mesma finalidade também apontou esta limitação (20).

O cuidado de Enfermagem no perioperatório das cirurgias geradoras de estomias intestinais de eliminação exige do enfermeiro conhecimento sobre recomendações pré e pós-operatórias baseadas em evidências ${ }^{(12-13)}$ capazes de fundamentar a tomada de decisão profissional e orientar a sua prática clínica.

No Brasil, estas recomendações ainda não existem e no Estado do Piauí, região nordeste do país, local onde este estudo foi realizado, por enquanto, há um Curso de Especialização em Enfermagem em Estomaterapia credenciado recentemente e o número de enfermeiros Estomaterapeutas é reduzido. Estes fatores podem especificamente nesta localidade do país prejudicar a qualidade do cuidado de Enfermagem que deve ser prestado às pessoas com estomias intestinais de eliminação e exigir por parte das instituições de ensino superior maior investimento na formação e capacitação de enfermeiros para aumentar o seu conhecimento e sua experiência, prevenindo complicações e promovendo o autocuidado desta população ${ }^{(5,10,21)}$.

Observa-se nos clientes orientados por enfermeiros no pré-operatório melhor adaptação ao estoma e a nova condição de $v i^{(3)}{ }^{(3)}$. Neste contexto, o enfermeiro deve ter suprimentos disponíveis para manutenção das habilidades necessárias ao cuidado de pessoas com estomas intestinais de eliminação e no mínimo ser capaz de realizar a troca de um equipamento coletor e ensinar estas habilidades para a pessoa recém estomizada ${ }^{(5)}$
Nota-se que, os itens do instrumento estão principalmente relacionados à assistência de Enfermagem no pré e pós-operatório (imediato, mediato e tardio) das estomias intestinais de eliminação, etapas que exigem do enfermeiro conhecimento de recomendações científicas ${ }^{(12,13)}$ para a prestação de cuidado seguro e efetivo.

A maioria dos itens obteve Índice de Validade de Conteúdo maior que $80,0 \%$ e apenas um item do pós-operatório imediato e outro do pós-operatório mediato obtiveram Índice de Validade de Conteúdo menor que 80,0\%. De acordo com a literatura itens com Índice de Validade de Conteúdo abaixo de $80,0 \%$ podem ser modificados ou excluídos ${ }^{(16)}$, neste estudo, a opção foi excluir. Recomendações pré-operatórias focalizam: educação de clientes e familiares sobre a estomia, demarcação do estoma de todos os clientes, preparação psicológica e exploração do possivel impacto da estomia sobre a sexualidade do estomizado. E pós-operatórias: avaliação imediata das condições do estoma e da pele periestomia e educação de clientes e familiares sobre o tipo de estomia, troca do equipamento coletor, alimentação, vestuário, possiveis alterações da imagem corporal e da intimidade, complicações comuns e recursos disponiveis, por exemplo, as políticas públicas e associações nacionais e locais de apoio aos estomizados ${ }^{(12-13)}$.

O instrumento construído e validado neste estudo pode ser uma ferramenta importante para mensurar o conhecimento de alunos de Graduação em Enfermagem sobre o cuidado de Enfermagem nas cirurgias geradoras de estomias intestinais de eliminação, contribuindo com o ensino e a assistência de Enfermagem.

\section{Limitações do estudo}

Como limitação, destaca-se o tipo de validação utilizada neste estudo. Ressalta-se a importância de estudos posteriores para a realização de outros tipos de validação.

\section{Contribuições do estudo para a prática}

Como contribuição, no Brasil, a aplicação do instrumento evidenciará as lacunas do conhecimento existentes sobre o cuidado de Enfermagem no perioperatório das cirurgias geradoras de estomias intestinais de eliminação, que poderão ser sanados mediante formação baseada em evidências científicas nos Cursos de Graduação em Enfermagem, Cursos de Especialização em Enfermagem em Estomaterapia ou em Programas de educação permanente para profissionais que estão na prática clínica.

\section{CONCLUSÃO}

Em conclusão, foi possível realizar a construção e validação de conteúdo do instrumento de avaliação do conhe- 
cimento de alunos de Graduação em Enfermagem e Enfermeiros sobre o cuidado de Enfermagem no perioperatório das cirurgias geradoras de estomias intestinais de eliminação.

\section{Contribuição dos autores}

Concepção e/ou desenho: Monteiro AKC, Campos MOB e Andrade EMLR; Análise e interpretação dos dados: Monteiro AKC, Campos MOB, Andrade EMLR e Andrade JX; Redação do artigo: Monteiro AKC, Campos MOB e Andrade EMLR; Revisão crítica: Monteiro AKC, Campos MOB, Andrade EMLR e Andrade JX; Revisão final: Monteiro AKC. 


\section{REFERÊNCIAS}

1. Goldberg M. Colwell J, Burns S, Carmel J. Fellows J. Hendren S et al. WOCN Society Clinical Guideline: Management of the Adult Patient with a fecal or urinary ostomy- an executive summary. JWound Ostomy Continence Nurs. [Internet]. 2018 [cited 2019 Jan 09]; 45 (1): 50-58. Available from: https://www.ncbi.nlm.nih.gov/pubmed/29300288.

2. Cengiz B, Bahar Z. Perceived Barriers and Home Care Needs When Adapting to a Fecal Ostomy: A Phenomenological Study. J Wound Ostomy Continence Nurs.[Internet]. 2017 [cited 2019 Jan 09]; 44(1):6368. Available from: https://www.ncbi.nlm.nih.gov/pubmed/27564927.

3. Oliveira LN, Lopes APAT, Decesaro MN. Cuidado integral à pessoa estomizada na atenção básica - conhecimento e atuação do enfermeiro. Ciência, Cuidado e Saúde [Internet]. 2017 [cited 2017 Jul 15]; 16 (3):1-8. Available from: http://www.periodicos.uem.br/ojs/index. php/CiencCuidSaude/article/view/35998.

4. Cross HH, Roe CA, Wang D. Staff nurse confidence in their skills and knowledge and barriers to caring for patients with ostomies. J Wound Ostomy Continence Nurs. [Internet]. 2014 [cited 2014 Dec 20]; 41 (6): 560-5. Available from: https://www.ncbi.nlm.nih.gov/ pubmed/25377106

5. Camargo JD, Raquel Motta RA.Viabilidade do Ensino Padronizado no Conteúdo de Estomaterapia na Graduação de Enfermagem: Proposta de Objeto de Aprendizagem Online. Rev Estima [Internet]. 2016 [cited 2018 Jul 16]; 14 (3): 109-117. Available from: https://www.revistaestima. com.br/index.php/estima/article/view/406.

6. Santos VLCGS, Cesaretti IUR. Assistência em Estomaterapia cuidando de pessoas com estomia. $2^{a}$ ed. Rio de Janeiro: Atheneu; 2015. 624p

7. Associação brasileira de estomaterapia - SOBEST. Estomaterapia - cursos de especialização em estomaterapia no Brasil. SOBEST [Internet]. 2017 [cited 2018 Jul 16]. Available from: http://www.sobest. org.br/texto/7.

8. Nafees B, Rasmussen M, LLoyd A.The Ostomy-Q: development and psychometric validation of an instrument to evaluate outcomes associated with ostomy appliances. Ostomy Wound Manage [Internet]. 2017 [cited 2018 Jul 17]; 63(1): 12-22. Availale from: https://www.ncbi. nlm.nih.gov/pubmed/28112646.

9. Farias RCM, Souza NVDO, Gonçalves FGA, David HMSL, Pires AS, Amorim LKA. O conhecimento dos enfermeiros residentes sobre 0 cuidado à pessoa com estomia intestinal. Rev. enferm. UERJ [Internet]. 2015 [cited 2019 Jan 11]; 23 (5): 656-6l. Availale from: https:// www.e-publicacoes.uerj.br/index.php/enfermagemuerj/article/ view/17966/15614

10. Zimnicki K, Pieper B. Assessment of Prelicensure Undergraduate Baccalaureate Nursing Students: Ostomy Knowledge, Skill Experiences, and Confidence in Care. Ostomy Wound Manage. 2018 [cited 2019 Jan 09]; 64 (8):35-42. Available from: https://www.ncbi.nlm. nih.gov/pubmed/30212363.
11. Bagheri M. Sharifan P. Far AB, Pouresmail Z, Kavousi F. Nurses knowledge about fecal intestinal ostomies's care: a cross-sectional study. Jundishapur J Chronic Dis Care [Internet]. 2016 [cited 2018 Jul 16]; 6 (2): e43345. Available from: http://jjchronic.com/en/ articles/14212.html.

12. Burch J. Stoma care: an update on current guidelines for community nurses. British Journal of Community Nursing [Internet]. 2017 [cited 2017 Dec 10]; 22(4):162-6. Available from: https://www.ncbi.nlm.nih. gov/pubmed/28414541.

13. Miller D. Pearsall E, Johnston D. Frecea M, McKenzie M. Enhanced recovery after surgery: best practice guideline for the care of patients with a fecal diversion. J Wound Ostomy Continence Nurs. [Internet]. 2017 [cited 2017 Dec 10]; 44(1):74-7. Available from: https://www.ncbi. nlm.nih.gov/pubmed/27163680

14. Paula MAB, Paula PR, Cesaretti, IUR. Estomaterapia em foco e o cuidado especializado. 1ạed. São Paulo: Yendis; 2014

15. Polit DF, Beck CT. Essentials of nursing research: appraising evidence for nursing practice. 9aed. Philadelphia: Wolkes Kluwer Health; 2018

16. Pasquali L. Instrumentação psicológica: fundamentos e práticas. $1^{a ̣ a}$ ed. Porto Alegre: Artmed; 2010

17. McGartland D, Marla Berg-Weger R, Tebb SS, Lee S, Rauch S. Objectifying content validity: Conducting a content validity study in social work research. Social Work Research [Internet]. 2003 [cited 2014 Dec 29]; 27 (2): 94-104. Available from: https://academic.oup.com/ swr/article-abstract/27/2/94/1659075?redirectedFrom=fulltext.

18. Fleiss JL. Measurihttp://psycnet.apa.org/fulltext/1972-05083-001 pdfng nominal scale agrément among many raters. Psycological Bulletin [Internet]. 1971[cited 2014 Dec 20]; 76(5): 378-382. Available from: http://psycnet.apa.org/fulltext/1972-05083-001.pdf.

19. Randolph JJ. Online Kappa Calculator. [Computer software]. 2008 [cited 2014 Dec 29]. Available from: http://justus.randolph.name/ kappa.

20. Pedreira RBS, Rocha SV, Santos CA, Vasconcelos LRC, Reis MC Content validity of the Geriatric Health Assessment Instrument Einstein [Internet]. 2016 [cited 2019 Jan 11]; 14(2):158-77. Available from: http://www.scielo.br/pdf/eins/v14n2/pt_1679-4508-eins-14-2-0158. pdf

21. Farias DLS, Nery RNB, Santana ME. O enfermeiro como educador em saúde da pessoa estomizada com câncer colorretal. Enferm Foco [Internet]. 2018 [cited 2019 Ago 08]. 10 (1): 35-39. Available from: http:// revista.cofen.gov.br/index.php/enfermagem/article/view/1486/490 